\title{
VIEWPOINT
}

\section{Selftrapping, biomolecules and free electron lasers}

\author{
Per-Anker Lindgård ${ }^{1,2}$ and A Marshall Stoneham ${ }^{3}$ \\ ${ }^{1}$ Materials Research Department, Ris $\emptyset$ National Laboratory, DK-4000 Roskilde, Denmark \\ ${ }^{2}$ QUP Centre, Danish Technical University, DK-2800 Lyngby, Denmark \\ 3 Department of Physics and Astronomy, University College London, Gower Street, \\ London, WC1E 6 BT, UK
}

Received 1 April 2003

Published 28 April 2003

Online at stacks.iop.org/JPhysCM/15/V5

For a solid-state physicist, the controlled movement of energy and charge are central phenomena. Novel materials have often pointed to new mechanisms: self-trapping in halides giving energy localization, the incoherent motion of small polarons in colossal magnetoresistance oxides, solitons in conducting polymers, magnetic polarons in magnetic semiconductors, the quantum motion of electrons in mesoscopic metals, the regimes of both coherent and incoherent quantum propagation of muons in solids, and so on. The issues of energy and charge transport are equally crucial in living matter, but are understood less well. How can the energy from light or chemical processes be moved around and used efficiently? The important paper by Austin et al (2003) in this special issue shows that new experimental tools, using the ultra short, tunable laser pulses from free electron lasers, open up the possibility of resolving outstanding problems in understanding the energy and charge processes which underpin life itself. In biological physics, one of the most intriguing ideas is that of the soliton, whose coherent motion is believed to enable efficient energy transport. Here we shall discuss the connection between these ideas of solitons and similar concepts occurring in more conventional condensed matter systems.

Light is the most important source of energy for living matter, and the interaction with light is of vital importance in many other circumstances. Yet, the optical properties of simple bio molecules - or even of simpler organic chemical analogues - are not fully understood. Is it true that, in living matter, energy (whether its origin is from light or from a chemical process) becomes localized, trapped in soliton modes, and subsequently transported deterministically in energy packets? This has been discussed widely in the 25 years since Davydov (1977) proposed that this might be what happens in the $\alpha$-helices in proteins. However, the idea is unproven. There has been no 'smoking gun' proving the hypothesis for real proteins, as Austin phrased it in his talk presenting the subject to be highlighted here. It is probably fair to say that there are two main reasons why interest in the Davydov model has been declining. First, it seems uncertain that solitons would be stable in proteins at physiological temperatures. Secondly, evidence for solitons has been at best circumstantial, based on the occurrence of temperature-dependent side-bands to certain peaks in the vibrational response spectrum. The problem is that the temperature dependence reflects the mean square deviation $\left\langle x^{2}\right\rangle$ of the atoms involved, and hence it is the same for static defects as for dynamic and nonlinear soliton 'defects' (Alexander and Krumhansl 1986); we remark further that $\left\langle x^{2}\right\rangle$ is not very sensitive to 


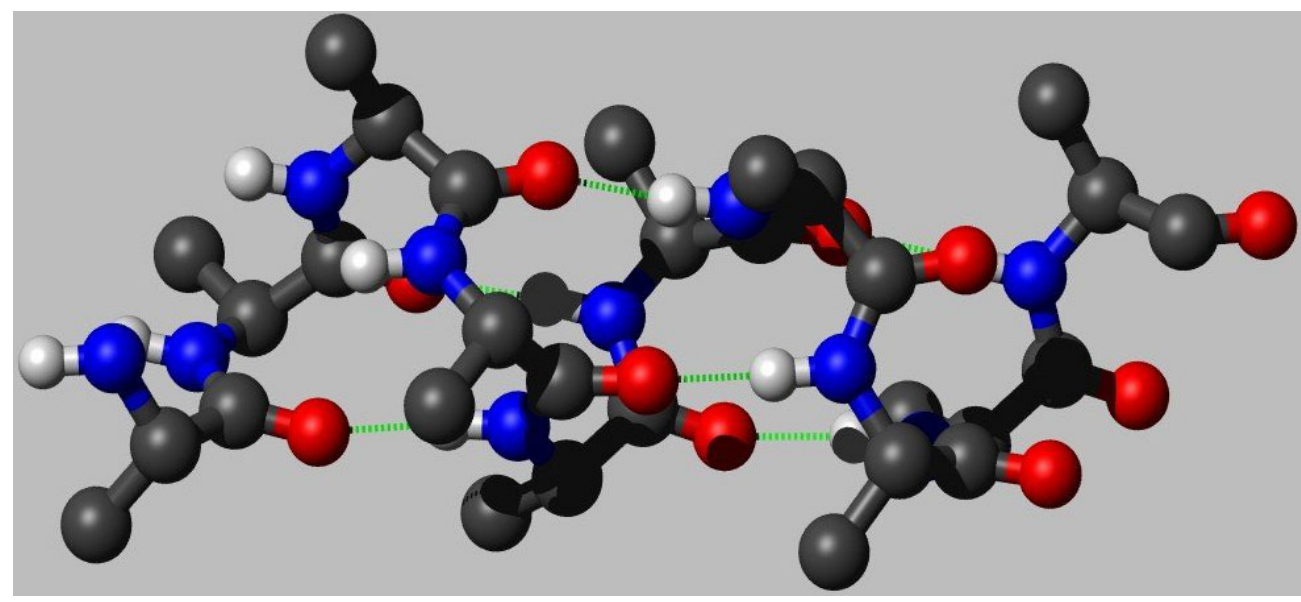

Figure 1. Portion of an $\alpha$-helical protein. The black, spiralling backbone is stabilized by hydrogen bonds, shown as green dashed lines. A number of the key $\mathrm{H}-\mathrm{N}-\mathrm{C}=\mathrm{O}$ peptide units can be identified. The amide-I vibration gives a very characteristic resonance, and is dominated by a $\mathrm{C}=\mathrm{O}$ stretch motion. $\mathrm{O}$ atoms are red, $\mathrm{H}$ atoms silver, $\mathrm{N}$ atoms blue, and $\mathrm{C}$ atoms black, where the outwardspointing ones indicate the beginning of usually different side-chains. These are attached to the $\mathrm{C} \alpha$ atoms, which are bound to the $\mathrm{N}$ and $\mathrm{C}$ in the peptide unit and form a flat, strongly bound platelet.

the details of the phonon spectrum (Stoneham 1975, p 316, Housely and Hess 1966, Johnson and Kassman 1969). Notice that independent measurements of $\left\langle x^{2}\right\rangle$ of specific atoms are presented in this special issue using neutron scattering (Zaccai 2003) and using Mössbauer spectroscopy (Achterhold and Parak 2003). The intensity of the response function (infrared absorption, Raman scattering, etc) should simply follow an $\exp \left(-(T / \theta)^{2}\right)$ dependence, where $T$ is the temperature and $\theta$ a constant.

The excitation of interest could be the $\mathrm{C}=\mathrm{O}$ stretch frequency (typically near $v \sim$ $1650 \mathrm{~cm}^{-1}=205 \mathrm{meV}=6.06 \mu \mathrm{m}=49.5 \mathrm{ps}^{-1}$ in the various units used preferentially in different fields) of the hydrogen bonded peptide chain units $\cdots \mathrm{H}-\mathrm{N}-\mathrm{C}=\mathrm{O}: \mathrm{H}-\mathrm{N}-\mathrm{C}=\mathrm{O} \cdots$ along the $\alpha$-helices (see figure 1). The frequency of that mode could be lowered if the local potential is softened due to a local relaxation of the surrounding atom positions; this might be due to a broken hydrogen bond giving a larger $\mathrm{O}-\mathrm{H}$ distance. Hence a red-shifted sideband is expected, corresponding to a non-linearly coupled mode pair. And the partner could be a low frequency, localized (soliton) excitation on the hydrogen bonded chains (the bonds indicated by the dotted lines in figure 1) stabilizing the $\alpha$-helices. However, the spectrum for a regular protein containing a large number $N$ of atoms (typically $N \sim$ thousands) is very complex. There will be at least $3 N$ response peaks corresponding to the $3 N$ harmonic eigenfrequencies. Then there will be higher harmonics, and the various non-linear mode combinations. So the reliable assignment of a particular peak is very difficult.

What would be the experimental signatures of such soliton modes or of the so-called selftrapping? The most distinct signature should be the frequency response of the soliton itselfthat could be the 'smoking gun'. Surprisingly, that signature was found in 1D magnetic systems as a peak centred on zero frequency, where no peak would be expected in a harmonic system. This has been much studied and discussed (Steiner et al 1976). For proteins there are many low frequency excitations corresponding to large scale vibrations and a similar detection is extremely difficult. So what is new? Recent time-resolved spectroscopic measurements allow one to probe the lifetime of particular, chosen excitations. Long relaxation times suggest 
soliton character and self-trapping. Using the possibilities of the free electron laser FELIX in the Netherlands, Austin et al have studied a number of such sidebands, finding unexpectedly long lifetimes $\sim 30$ ps as opposed to only 5 ps for the neighbouring peaks. That is very interesting because it indicates that the energy is localized and not easily dispersed in the full protein. The sideband excitation can hence act as an energy reservoir. Studies of real proteins indicate long-lived states in sperm whale myoglobin (Xie et al 2000) as in bacteriorhodopsin (Austin et al 2003), both mainly consisting of long $\alpha$-helices. A peak in the far infrared end (at $v \sim 115 \mathrm{~cm}^{-1}$ ) in bacteriorhodopsin has unexpectedly long relaxation times $\sim 500 \mathrm{ps}$ (Xie et al 2002). D'Ovidio et al (2003) demonstrate that this mode is probably associated with excitations on the hydrogen bonds along the sides of the $\alpha$-helices, and these have soliton character, as well as minimal effect on the surrounding protein and solvent. Hence, the mode has all the properties needed for being long-lived in comparison to other low frequency modes involving large-scale fluctuations of the whole protein. Austin et al associate the temperature dependent sideband observed in the static IR spectrum with the long lived mode, but are cautious in describing it as a 'self-trapped' excitation. In particular they demonstrate that the temperature dependence is consistent with a simple two-state model-and do not use that expected for the self-trapping model. A two state model was also used by Williams et al (1996), who investigated a small artificial 21-residue $\alpha$-helical peptide by time-resolved IR spectroscopy. They suggest the temperature dependence is due to broken hydrogen bonds (or a helix-coil transition) at high temperatures, yet without fully destroying the $\alpha$-helix architecture. This is a simple picture, which may or may not be the same, physically, as provided by the self-trapping theory. Clearly, a great deal is to be learned from the new possibilities opened up by ultra fast time-resolved experiments.

At this point, it is appropriate to seek comparison with the longer-established ideas of conventional condensed matter systems with impurities or other imperfections. In such systems, light is absorbed and stored in excited states. These states are localized, and are separated in frequency from other modes (phonons or electronic) in the crystal (Barker and Sievers 1975, Stoneham 1975). There are various states (see below) in which electrons are strongly coupled to host lattice motion (Shluger and Stoneham 1993, Shluger et al 2000). These include behaviour associated with fluctuations of potentials and force constants due to thermal vibrational motions. This may lead to a local 'dynamic defect'. This is interesting if it happens on the time scale similar to that of the self-trapping relaxation. In anharmonic systems different (harmonic) excitations couple, when such a dynamic defect might trap another excitation. This is not necessarily a one-dimensional problem.

One finds a number of related, thought provoking terms and concepts in both condensed matter physics and in the biological physics problem discussed above. Since the terms and the concepts do not always correspond precisely, it may be useful in this viewpoint article to mention concisely a few of the most important ideas in the form of a 'dictionary with comments'. Many more examples could be mentioned in a full-length review.

The first set of ideas relate to the localization and transport of charge. One important point is that those carriers which interact with the lattice (e.g., polarons) are less mobile than carriers without interaction. So the effects might be the opposite of what Davydov wanted his soliton to do.

(1) Soliton: a soliton in biomolecules offers one possible way to understand how energy released by ATP hydrolysis can be propagated over a fairly long distance (maybe $150 \mathrm{~nm}$ ) without significant dissipation. So the idea is that there is a motion (coherent in some sense) enabled by an anharmonic interaction between conventional vibrations and the $\mathrm{C}=\mathrm{O}$ mode (Davydov 1977). This corresponds to the solitons of applied mathematics and shares their 
properties in propagating roughly as desired (Scott 1992). In condensed matter physics, solitons are commonly discussed for conjugated polymers (semiconducting polymers like trans-polyacetylene, t-PA) and in the propagation of light down optical fibres. The polymer solitons are very like polarons (and indeed, the two words are used for similar excitations differing mainly in topology). Models of t-PA (Su et al 1980, Wallace et al 1991) show solitons propagating at just below the speed of sound. The idea is really rather similar to the biomolecule case, but, instead of transporting a $\mathrm{C}=\mathrm{O}$ vibration, the coupling is due to the dimerization. On the borderline is the 'biomolecular-like' crystal of acetanilide, which has the same hydrogen bonded peptide chain units as found in $\alpha$-helices. A recent pump-probe experiment discusses the complex sidebands of the $\mathrm{N}-\mathrm{H}$ stretch vibration in terms of the self-trapping mechanism (Edler et al 2002).

(2) Polaron: in the conventional condensed matter case, there are two types of polarons: large and small (Stoneham 1989). Both concern carriers (electrons or holes) and the associated strain and polarization they cause in the host solid. Almost all the theory is harmonic: there is no need for anharmonicity. A large polaron means a carrier plus its associated strain/polarization field, and perhaps the main effect of this field is to alter the effective mass of the carrier. So, for example, its mobility falls as temperature rises, because of scatter by increasing vibrational amplitudes. A small polaron (using the conventional view; there are subtleties) is self-trapped: the distortion or polarization is so large as to immobilize the carrier (that was first shown by Landau 1933).

(3) Self-trapping charge localization: the small polaron moves by incoherent hopping, so its mobility increases as temperature rises (again, there are qualifications, and for muons in solids there is another regime at low temperatures when the scattering vibrations are not excited). Self-trapping localizes the carrier, so gives charge localization (Itoh and Stoneham 2000). The localized carrier can be observed spectroscopically. The selftrapped hole $\left(\mathrm{V}_{\mathrm{k}}\right.$ centre $)$ in halides has been studied extensively both optically and in spin resonance.

(4) Self-trapping energy localization: excitons can also self-trap. Sometimes the result can be considered as an electron trapped by a self-trapped hole (or vice versa); sometimes the self-trapped exciton has quite a different structure. Exciton self-trapping gives energy localization and the localized energy can cause local atomic changes and defect production. In principle, excitons can transport energy (create an exciton at site 1, let it move to site 2, recombine to release energy).

(5) Localized vibrations: in solids have two main forms. Local modes have frequencies different from those of the host, whether at higher frequencies (the common case for light impurities) or in a gap in the phonon density of states. Resonant modes concentrate vibrational energy at specific sites, often at heavy impurities. However, some impurities (e.g., $\mathrm{KCl}: \mathrm{Li})$ show local modes, resonant modes, and even tunnelling states.

How were the electronic and excitonic self-trapped states identified? Spectroscopy has been especially powerful. For example, the self-trapped hole in $\mathrm{KCl}$ might be regarded as a $\mathrm{Cl}_{2}^{-}$molecular ion replacing two $\mathrm{Cl}^{-}$ions, and it has characteristic spin resonance, Raman, optical and infrared transitions. The local distortion has observable effects. One might ask whether optical or spin resonance spectra, rather than vibrational spectroscopies, could yield useful information on possible Davydov soliton systems. Some steps in this direction are found in pump probe experiments, which test some of the key ideas by exploiting the possibilities of tunable lasers offering ultra fast pulses. Several examples of such experiments are presented in the paper by Schoenlein et al (2000) in which the technique is described. The temperature dependences and lifetimes of selected excitations can now be measured using modern pump- 
probe techniques. Such properties are also highly relevant in more conventional condensed matter systems (Itoh and Stoneham 2000).

The recent novel results for real proteins are bringing new insight to the dynamical and optical properties of living matter, and provide a substantial step towards understanding their function. It is striking that the extremely high time resolution, typically femtoseconds, is an essential ingredient for insight into phenomena at much longer times scales, typically nanoseconds to microseconds for proteins. There are significant parallels between the concepts for proteins and those for inorganic solids, and there are signs that applying the full range of experimental tools would transform our understanding of both classes of system. This would provide a powerful array of ideas and approaches, and perhaps a route to understanding those many systems which involve the sophisticated dynamical behaviour of soft and hard matter.

\section{Acknowledgments}

We thank Dr Geoffrey Wells and Dr Sarah Harris for the figure.

\section{References}

Achterhold K and Parak F G 2003 J. Phys.: Condens. Matter 151683

Alexander D M and Krumhansl J A 1986 Phys. Rev. B 337172

Austin R H, Xie A, van der Meer L, Shinn M and Neil G 2003 J. Phys.: Condens. Matter 151693

Barker A S and Sievers A J 1975 Rev. Mod. Phys. 47 Suppl no 2

Davydov A S 1977 J. Theor. Biol. 66377

Davydov A S 1979 Phys. Scr. 20387

D’Ovidio F, Lindgård P A and Bohr H G 2003 J. Phys.: Condens. Matter 151699

Edler J, Hamm P and Scott A C 2002 Phys. Rev. Lett. 88 067403-1

Housely R M and Hess F 1966 Phys. Rev. 146517

Itoh N and Stoneham A M 2000 Materials Modification by Electronic Excitation (Cambridge: Cambridge University Press) $\mathrm{p} \mathrm{xv}$ and 520

Johnson D P and Kassman A J 1969 Phys. Rev. 1881385

Landau L D 1933 Phys. Z. 3664

Schoenlein R W, Chattopadhyay S, Chong H H W, Glover T E, Heimann P A, Shank C V, Zholents A A and Zolotorev M S 2000 Science 2872237

Scott A C 1992 Phys. Rep. 2171

Shluger A L, Gavartin J L, Szymanski M A and Stoneham A M 2000 Nucl. Instrum. Methods Phys. Res. B 166/167 1

Shluger A L and Stoneham A M 1993 Small polarons in real crystals: concepts and problems J. Phys.: Condens. Matter 1 3049-86

Steiner M J, Villain J and Windsor C G 1976 Adv. Phys. 2587

Stoneham A M 1975 Theory of Defects in Solids (Oxford: Oxford University Press)

Stoneham A M 1989 Small polarons and polaron transitions J. Chem. Soc. Faraday II 85 505-16

Su W P, Schrieffer J R and Heeger A J 1980 Phys. Rev. B 222099

Wallace D S, Stoneham A M, Hayes W, Fisher A J and Testa A 1991 J. Phys.: Condens. Matter 33905

Williams S, Causgrove T P, Gilmanshin R, Fang K S, Callender R H, Woodruff W H and Dyer R B 1996 Biochemistry 35691

Xie A, van der Meer A F G and Austin R H 2002 Phys. Rev. Lett. 88 018102-1

Xie A, van der Meer L, Hoff V and Austin R H 2000 Phys. Rev. Lett. 845435

Zaccai G 2003 J. Phys.: Condens. Matter 151673 